\title{
More Transparency in BioAnalysis of Exocytosis: Coupling of Electrochemistry and Fluorescence Microscopy at ITO Electrodes
}

\author{
Xiaoqing Liu, Damien Quinton, Lihui Hu, Christian Amatore, Jérôme Delacotte, Frédéric Lemaître, \\ Manon Guille-Collignon ${ }^{a}$ \\ Ecole Normale Supérieure-PSL Research University, Département de Chimie, Sorbonne Universités - UPMC Univ \\ Paris 06, CNRS UMR 8640 PASTEUR, 24, rue Lhomond, 75005 Paris, France
}

\begin{abstract}
Vesicular exocytosis is an essential biological mechanism used by cellular organisms to release bioactive molecules (hormones, neurotransmitters...) in their environment. For instance, this is the pathway by which chromaffin cells deliver catecholamines (adrenaline, nor-adrenaline, dopamine...) in blood. During this process, secretory vesicles that initially stored the (bio)chemical messengers dock to the cell membrane. The subsequent fusion of vesicle and cell membranes induces the formation of a fusion pore that initiates the first exchanges between the intravesicular and extracellular media. Its following expansion thus favours a larger release of the vesicular content into the external medium. Several analytical methods have been developed in order to study exocytosis at the single living cell level in real time. Among those techniques, mostly based on electric or optic measurements, amperometry with a carbon-fiber ultramicroelectrode [1], used in the first part of this report, and total internal reflection fluorescence microscopy (TIRFM) appear as the most powerful [2] Practically, physico-chemical properties of ultramicroelectrodes induce a high detection sensitivity and temporal resolution, thus being particularly well adapted to monitor exocytosis of electroactive molecules in real time.
\end{abstract}

In this work, exocytosis is first studied amperometrically (at carbon fiber ultramicroelectrodes) at adrenal chromaffin cells, which release catecholamines after appropriate stimulation, while testing the effects due to trans-insertion of two exogenous compounds (lysophosphatidylcholine (LPC) and arachidonic acid (AA)) on the kinetics of exocytotic events. Amperometric analyses showed that, under the present conditions (short incubation times and micromolar LPC or AA solutions), LPC favors catecholamine release (rate, event frequency, charge released) while AA disfavors the exocytotic processes. We also observed that the detected amount of neurotransmitters in the presence of LPC was larger than under control conditions, while the opposite trend is observed with AA [3].

Nevertheless, amperometric investigations are obviously limited to the phenomena occurring once the fusion pore onsets. Coupling this technique with fluorescence microscopy would permit to

${ }^{\text {a }}$ Corresponding author: manon.guille@ens.fr 
visualize the status of vesicles near the plasma membrane, more particularly their behaviour before and during the fusion as well as the location of the event. In this regard, the combination has to involve a cellular model whose secretory vesicles are both marked by a fluorescent probe and whose content is electroactive. To do so, amperometric and optical investigations have been achieved here to choose a cell model for which the features of the exocytotic events are sufficient to possibly correlate the electrochemical and optical signals. Moreover, the amperometry-microscopy combination also requires the development of specific devices. Thus, semiconducting and transparent ITO (Indium Tin Oxide) is used as an electrode without hindering the optic detection of marked vesicle by a fluorescent probe. Feasibility of this coupling strategy will be demonstrated and results obtained in these conditions on single cell will be described and discussed [4-7].
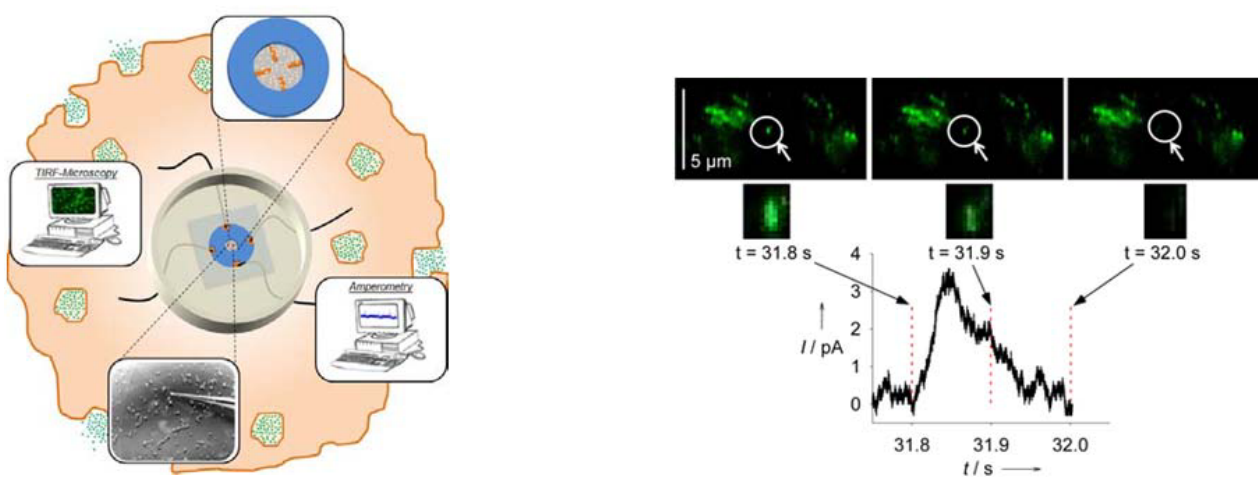

Figure 1. Example of a combined detection of exocytosis on enterochromaffin BON cells. Left: experimental set-up. Right: Optical and electrical combined detection of a single exocytotic event.

\section{References}

1. C. Amatore, S. Arbault, M. Guille, F. Lemaître Chem Rev 108, 2585 (2008)

2. E. Karatekin, V. S. Tran, S. Huet, I. Fanget, S. Cribier, J-P. Henry Biophys. J. 94, 2891 (2008)

3. L. Chernomordik, A. Chanturiya, J. Green, J. Zimmerberg Biophys. J. 69, 922 (1995) ; C. Amatore, S. Arbault, Y. Bouret, M. Guille, F. Lemaître, Y. Verchier ChemBioChem 7, 1998 (2006)

4. A. Meunier, O. Jouannot, R. Fulcrand, I. Fanget, M. Bretou, E. Karatekin, S. Arbault, M. Guille, F. Darchen, F. Lemaître, C. Amatore Angew. Chem. 50, 5081 (2011)

5. A. Meunier, R. Fulcrand, F. Darchen, M. Guille-Collignon, F. Lemaître, C. Amatore Biophys. Chem. 162, 14 (2012)

6. A. Meunier, M. Bretou, F. Darchen, M. Guille-Collignon, F. Lemaître, C. Amatore Electrochimica Acta 126, 74 (2014)

7. F. Lemaître, M. Guille-Collignon, C. Amatore Electrochimica Acta 140, 457 (2014) 